\title{
BARTLETT SPECTRUM AND MIXING PROPERTIES OF INFINITELY DIVISIBLE RANDOM MEASURES
}

\author{
EMMANUEL ROY, ${ }^{*}$ Université Paris 13
}

\begin{abstract}
We prove that the Bartlett spectrum of a stationary, infinitely divisible (ID) random measure determines ergodicity, weak mixing, and mixing. In this context, the Bartlett spectrum plays the same role as the spectral measure of a stationary Gaussian process.

Keywords: Infinitely divisible random measure; infinitely divisible point process; Cox process; Bartlett spectrum; ergodic theory
\end{abstract}

2000 Mathematics Subject Classification: Primary 60G57; 60G55

Secondary 37A05; 60E07

\section{Introduction}

Random measures, and particularly point processes, are widely used in stochastic geometry and applied mathematics. ID point processes are surely the most commonly found point processes in the applications; see [5] and [7]. Poisson point processes, cluster Poisson processes, Cox processes with ID directing measure, Hawkes processes without ancestors (see [2]) among others, are ID point processes.

When stationarity, with respect to time (or to space in higher dimensions), is taken as an assumption, one is interested in the asymptotic independence properties known as mixing properties, which can be very difficult to determine. These properties are well characterised in terms of the KLM measure associated to any ID random measure (see [7]), however, this measure is rarely available. One quantity we often get access to, if a random measure $N$ is square integrable, is the Bartlett spectrum, which is the $\sigma$-finite measure $\Gamma$ defined by

$$
\operatorname{cov}[N(f), N(g)]=\int_{\mathbb{R}^{d}} \hat{f} \overline{\hat{g}} \mathrm{~d} \Gamma
$$

for all $f$ and $g$ in the Schwartz space, where $\hat{f}$ and $\hat{g}$ are the Fourier transforms of $f$ and $g$ (see [4, Chapter 11] for details and examples). The Bartlett spectrum is a useful tool to study second-order properties of a random measure. The aim of this paper is to show that a square integrable stationary ID random measure is:

- ergodic (and weakly mixing) if and only if $\Gamma\{0\}=0$,

- mixing if and only if $\Gamma^{\prime}$ is Rajchman, that is, if and only if $\int_{\mathbb{R}^{d}} \mathrm{e}^{\mathrm{i} t x} \Gamma^{\prime}(\mathrm{d} x) \rightarrow 0$ as $\|t\| \rightarrow \infty$

where $\Gamma^{\prime}$ denotes any finite measure equivalent to $\Gamma$ (the result does not depend on the choice of $\left.\Gamma^{\prime}\right)$.

Received 2 February 2007; revision received 27 September 2007.

* Current address: Laboratoire Analyse, Géométrie et Applications (LAGA), Université Paris 13, UMR 7539, 99 avenue

J. B. Clément, F-93430 Villetaneuse, France. Email address: roy@ math.univ-paris13.fr 
A stationary ID random measure is, thus, mixing as soon as its Bartlett spectrum is absolutely continuous with respect to the Lebesgue measure, which is the commonest situation in the applications. This also leads to a new proof of the mixing property for Hawkes processes without ancestors; see [2]. This result can be compared to the following well-known fact (see [3, p. 192]). A stationary Gaussian process $\left\{X_{t}\right\}_{t \in \mathbb{R}}$ is:

- ergodic (and weakly mixing) if and only if $\sigma_{X}$ is continuous,

- mixing if and only if $\sigma_{X}$ is Rajchman,

where $\sigma_{X}$ is defined by

$$
\operatorname{cov}\left[X_{0}, X_{t}\right]=\int_{\mathbb{R}} \mathrm{e}^{\mathrm{i} t x} \sigma_{X}(\mathrm{~d} x)
$$

The commonality between Gaussian processes and ID random measures is their infinite divisibility.

\section{The proof}

\subsection{Spectral preliminaries}

We recall some basic facts about spectral theory of unitary operators. If $(H,\langle\cdot, \cdot\rangle)$ is a complex, separable Hilbert space and $\left\{\Psi_{t}\right\}_{t \in \mathbb{R}^{d}}$ is a continuous group of unitary operators, the spectral measure of a vector $f \in H$ is the measure $\sigma_{f}$ on $\mathbb{R}^{d}$ uniquely defined by

$$
\left\langle\Psi_{t} \boldsymbol{f}, \boldsymbol{f}\right\rangle=\int_{\mathbb{R}^{d}} \mathrm{e}^{\mathrm{i} t s} \sigma_{f}(\mathrm{~d} s) .
$$

There exists a vector $f_{\mathrm{M}}$ such that, for any $\boldsymbol{f} \in H, \sigma_{f} \ll \sigma_{f_{\mathrm{M}}}$, where (the equivalence class of) $\sigma_{f_{\mathrm{M}}}$ is called the maximal spectral type of $\left\{\Psi_{t}\right\}_{t \in \mathbb{R}^{d}}$ on $H$.

If $\left(X, \mathcal{A}, m,\left\{T_{t}\right\}_{t \in \mathbb{R}^{d}}\right)$ is a $\sigma$-finite measure-preserving dynamical system, we consider the Hilbert space $L^{2}(m)$ and the group of unitary operators $\left\{W_{t}\right\}_{t \in \mathbb{R}^{d}}$ defined by $W_{t} f=f \circ T_{t}$.

If $m$ is a probability measure, the reduced maximal spectral type of $\left\{T_{t}\right\}_{t \in \mathbb{R}^{d}}$ is the maximal spectral type $\sigma_{\text {red }}$ of $\left\{W_{t}\right\}_{t \in \mathbb{R}^{d}}$ on $L_{0}^{2}(m):=L^{2}(m) \ominus \mathbb{C}$ (the subspace of zero-mean vectors). Weak mixing and mixing are spectral properties; see [3].

Theorem 2.1. The probability-preserving dynamical system $\left(X, \mathcal{A}, m,\left\{T_{t}\right\}_{t \in \mathbb{R}^{d}}\right)$ is weakly mixing if and only if $\sigma_{\mathrm{red}}$ is continuous, and mixing if and only if $\sigma_{\mathrm{red}}$ is Rajchman.

\subsection{The proof itself}

We first treat the case of point processes. The technique consists of obtaining our ID point process as a functional of a 'bigger' point process which is a Poisson measure (in this ergodic context, it is called Poisson suspension) with a well-chosen intensity. The Poisson suspension has a transparent spectral structure and is, thus, much more easier to study. Finally, we remark that the ergodic properties deduced at the level of the Poisson suspension are inherited by the initial ID point process.

Denote by $\left(M_{\mathbb{R}^{d}}, \mathcal{M}_{\mathbb{B}^{d}}\right)$ the canonical space of point processes on $\left(\mathbb{R}^{d}, \mathscr{B}^{d}\right)$. In the sequel $\mathrm{P}$ is always assumed to be stationary with respect to the natural flow of translations $\left\{\theta_{t}\right\}_{t \in \mathbb{R}^{d}}$. For any $A \in \mathscr{B}^{d}$ and $v \in M_{\mathbb{R}^{d}}, N(A)$ is defined by $N(A)(v)=v(A)$. Take a bounded set $A \in \mathcal{B}^{d}$, P is said to be square integrable if $\mathrm{E}\left[N(A)^{2}\right]<\infty$ and the Bartlett spectrum is well defined by (1.1). Thanks to the classical theory for ID point processes (see [7]), for 
any ID probability measure $\mathrm{P}$ on $\left(M_{\mathbb{R}^{d}}, \mathcal{M}_{\mathcal{B}^{d}}\right)$, there exists a uniquely determined $\sigma$-finite $\left\{\theta_{t}\right\}_{t \in \mathbb{R}^{d}}$-invariant measure $\mathrm{Q}$ on $\left(M_{\mathbb{R}^{d}}, \mathcal{M}_{\mathcal{B}^{d}}\right)$, called the KLM measure of $\mathrm{P}$, such that $\mathrm{Q}\left(\mu_{0}\right)=0$, where $\mu_{0}$ is the null measure on $\left(\mathbb{R}^{d}, \mathscr{B}^{d}\right)$, and such that, for all positive functions $f$,

$$
\mathrm{E}\left[\mathrm{e}^{-N(f)}\right]=\exp \int_{M_{\mathbb{R}^{d}}}\left(1-\mathrm{e}^{-\mu(f)}\right) \mathrm{Q}(\mathrm{d} \mu) .
$$

Now we form a new point process, the Poisson suspension over the system $\left(M_{\mathbb{R}^{d}}\right.$,

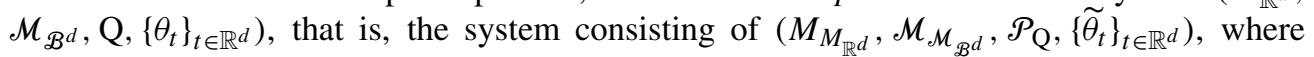
$\left(M_{M_{\mathbb{R}^{d}}}, \mathcal{M}_{\mathcal{M}_{\mathcal{B}^{d}}}\right)$ is the canonical space of point processes on $\left(M_{\mathbb{R}^{d}}, \mathcal{M}_{\mathcal{B}^{d}}\right), \mathcal{P}_{\mathrm{Q}}$ is the distribution of the Poisson measure of intensity Q, and, for all $t \in \mathbb{R}^{d}$ and $\tilde{\theta}_{t}$, the map $\gamma \rightarrow \gamma \circ \theta_{t}^{-1}$. It is easily checked that $\mathcal{P}_{\mathrm{Q}}$ is invariant under the action of $\left\{\tilde{\theta}_{t}\right\}_{t \in \mathbb{R}^{d}}$. Here $\mathfrak{N}(A)$ denotes the mapping $\mathfrak{N}(A)(\gamma)=\gamma(A)$ for $A \in \mathcal{M}_{\mathcal{B}^{d}}$ and $\gamma \in M_{M_{\mathbb{P} d}}$.

It is well known that $L^{2}\left(\mathcal{P}_{\mathrm{Q}}\right)$ can be identified with the Fock space $F\left[L^{2}(\mathrm{Q})\right]$ over $L^{2}(\mathrm{Q})$ :

$$
F\left[L^{2}(\mathrm{Q})\right]=\mathbb{C} \oplus L^{2}(\mathrm{Q}) \oplus \cdots \oplus\left[L^{2}(\mathrm{Q})\right]^{\odot n} \oplus \cdots,
$$

where $\left[L^{2}(\mathrm{Q})\right]^{\odot n}$ is the $n$th symmetric tensor power of $L^{2}(\mathrm{Q})$ and is called the $n$th chaos. The identification is made through multiple stochastic integrals; we only need to know what happens in the first chaos: if $f \in L^{1}(\mathrm{Q}) \cap L^{2}(\mathrm{Q})$ then $\left(\int_{M_{\mathbb{R}^{d}}} f \mathrm{~d} \mathfrak{N}-\int_{M_{\mathbb{R}^{d}}} f \mathrm{dQ}\right)$ in $L^{2}\left(\mathcal{P}_{\mathrm{Q}}\right)$ corresponds to $f$ in $L^{2}(\mathrm{Q}) \hookrightarrow F\left[L^{2}(\mathrm{Q})\right]$.

Let $\left\{U_{t}\right\}_{t \in \mathbb{R}^{d}}$ be the unitary operator associated to $\left\{\tilde{\theta}_{t}\right\}_{t \in \mathbb{R}^{d}}$ on $L^{2}\left(\mathcal{P}_{\mathrm{Q}}\right)$ defined by $U_{t} f=$ $f \circ \widetilde{\theta}_{t}$; let $\left\{V_{t}\right\}_{t \in \mathbb{R}^{d}}$ be the unitary operator associated to $\left\{\theta_{t}\right\}_{t \in \mathbb{R}^{d}}$ on $L^{2}(\mathrm{Q})$ defined by $V_{t} f=$ $f \circ \theta_{t}$. It is easy to see that $U_{t}$ acts as the $n$th tensor product of $V_{t}$ on the $n$th chaos. Thus, if $\sigma$ is the maximal spectral type of $\left\{V_{t}\right\}_{t \in \mathbb{R}}, \sum_{k=1}^{\infty}(1 / k !) \sigma^{* k}$ is the reduced maximal spectral type of $\left\{U_{t}\right\}_{t \in \mathbb{R}}$.

The crucial point here is that, thanks to an easy computation, we have a semiconjugation between $\left(M_{M_{\mathbb{R}^{d}}}, \mathcal{M}_{\mathcal{M}_{\mathcal{B}^{d}}}, \mathcal{P}_{\mathrm{Q}},\left\{\widetilde{\theta}_{t}\right\}_{t \in \mathbb{R}^{d}}\right)$ and $\left(M_{\mathbb{R}^{d}}, \mathcal{M}_{\mathcal{B}^{d}}, \mathrm{P},\left\{\theta_{t}\right\}_{t \in \mathbb{R}^{d}}\right)$, implemented by the map $\varphi$ from $M_{M_{\mathbb{R}^{d}}}$ to $M_{\mathbb{R}^{d}}$ :

$$
\gamma \mapsto \int_{M_{\mathbb{R}^{d}}} v(\cdot) \gamma(\mathrm{d} \nu),
$$

that is, $\mathrm{P}=\mathcal{P}_{\mathrm{Q}} \circ \varphi^{-1}$ and, for all $t \in \mathbb{R}^{d}, \varphi \circ \widetilde{\theta}_{t}=\theta_{t} \circ \varphi$. In terms of random variables, this means that we recover our ID point process $N$ of distribution $\mathrm{P}$ as a functional of the Poisson measure $\mathfrak{N}$ : for any $A \in \mathscr{B}^{d}, N(A)$ has the same distribution under $\mathrm{P}$ as $\int_{M_{\mathbb{R}^{d}}} v(A) \mathfrak{N}(\mathrm{d} v)$ under $\mathcal{P}_{\mathrm{Q}}$.

Now assume that $\Gamma\{0\}=0$ and assume that there exists a $\left\{\theta_{t}\right\}_{t \in \mathbb{R}^{d}}$-invariant set $B$ such that $0<\mathrm{Q}(B)<\infty$. The spectral measure of $\mathbf{1}_{B}$ on $L^{2}(\mathrm{Q})$ is $\mathrm{Q}(B) \delta_{\{0\}}$. Consider the map

$$
\mathcal{E}: x \rightarrow \exp \left(-\sum_{k=1}^{d}\left|x_{k}\right|\right) \quad \text { on } \mathbb{R}^{d},
$$

and let $\lambda$ be the Lebesgue measure.

The spectral measure of $\int_{\mathbb{R}^{d}} \varepsilon \mathrm{d} N-\int_{\mathbb{R}^{d}} \varepsilon \mathrm{d} \lambda$ on $L^{2}(\mathrm{P})$ is $\left(1 /\left(1+\|x\|^{2}\right)\right) \mathrm{d} \Gamma$, and thanks to the previous semiconjugation, is also the spectral measure of

$$
\int_{M_{\mathbb{R}^{d}}}\left(\int_{\mathbb{R}^{d}} \mathcal{E} \mathrm{d} \nu\right) \mathfrak{N}(\mathrm{d} \nu)-\int_{\mathbb{R}^{d}}\left(\int_{\mathbb{R}^{d}} \mathcal{E} \mathrm{d} \nu\right) \mathrm{d} Q
$$

on $L^{2}\left(\mathcal{P}_{\mathrm{Q}}\right)$ and, thus, also the spectral measure of $\int_{\mathbb{R}^{d}} \& \mathrm{~d} N$ on $L^{2}(\mathrm{Q})$. 
Since $\left(1 /\left(1+\|x\|^{2}\right)\right) \mathrm{d} \Gamma$ and $\mathrm{Q}(B) \delta_{\{0\}}$ are mutually singular, $\int_{\mathbb{R}^{d}} \mathcal{E} \mathrm{d} N$ and $\mathbf{1}_{B}$ are orthogonal, that is,

$$
\int_{M_{\mathbb{R}^{d}}}\left(\mathbf{1}_{B} \int_{\mathbb{R}^{d}} \mathscr{E} \mathrm{d} N\right) \mathrm{dQ}=0
$$

and this implies, since $\mathbf{1}_{B} \int_{\mathbb{R}^{d}} \varepsilon \mathrm{d} N \geq 0$, that

$$
\mathbf{1}_{B} \int_{\mathbb{R}^{d}} \mathcal{E} \mathrm{d} N=0 \quad \mathrm{Q} \text {-almost everywhere (Q-a.e.). }
$$

But $\int_{\mathbb{R}^{d}} \mathscr{E} \mathrm{d} N$ is strictly positive, thus, $\mathbf{1}_{B}=0$ Q-a.e. and $\mathrm{Q}(B)=0$, which is a contradiction. Then, there exists no $\left\{\theta_{t}\right\}_{t \in \mathbb{R}^{d}}$-invariant set of finite positive measure for $\left(M_{\mathbb{R}^{d}}, \mathcal{M}_{\mathcal{B}^{d}}, \mathrm{Q}\right.$, $\left\{\theta_{t}\right\}_{t \in \mathbb{R}^{d}}$. As in [1, p. 74], the absence of such a set implies that $\left\{V_{t}\right\}_{t \in \mathbb{R}^{d}}$ has no eigenvalue, which means that $\sigma$ is continuous.

We deduce that $\sum_{k=1}^{\infty}(1 / k !) \sigma^{* k}$ is also continuous and this proves that $\left(M_{M_{\mathbb{R}^{d}}}, \mathcal{M}_{\mathcal{M}_{\mathcal{B}^{d}}}, \mathcal{P}_{\mathrm{Q}}\right.$, $\left.\left\{\tilde{\theta}_{t}\right\}_{t \in \mathbb{R}^{d}}\right)$ is weakly mixing by Theorem 2.1 , a property which is inherited by $\left(M_{\mathbb{R}^{d}}, \mathscr{M}_{\mathcal{B}^{d}}, \mathrm{P}\right.$, $\left\{\theta_{t}\right\}_{t \in \mathbb{R}^{d}}$ ) through the semiconjugation.

Now assume that $\Gamma^{\prime}$ is Rajchman, where $\Gamma^{\prime}$ denotes a finite measure equivalent to $\Gamma$. As in the first part of the proof, we note that, for all bounded $A \in \mathcal{B}^{d}$, the spectral measure of $N(A)-\lambda(A)$ on $L^{2}(\mathrm{P})$ is also the spectral measure of $N(A)$ on $L^{2}(\mathrm{Q})$, and this measure is absolutely continuous with respect to $\Gamma^{\prime}$ and, thus, is also Rajchman. We can then observe that

$$
\mathrm{Q}\left(\{N(A)>0\} \cap \theta_{t}^{-1}\{N(A)>0\}\right) \leq \int_{M_{\mathbb{R}^{d}}} v(A) \nu(A) \circ \theta_{t} \mathrm{Q}(\mathrm{d} v) \rightarrow 0 \quad \text { as }\|t\| \rightarrow \infty .
$$

But, by applying the exhaustion lemma, as in [6], we can form the measurable union of the hereditary family of those sets $B \in \mathcal{M}_{\mathcal{B}^{d}}$ such that $0<\mathrm{Q}(B)<\infty$ and $\mathrm{Q}\left(B \cap \theta_{t}^{-1} B\right) \rightarrow 0$ as $\|t\| \rightarrow \infty$. And since the union of sets of the kind $\{N(A)>0\}$ for all bounded sets $A \in \mathscr{B}^{d}$ is the whole space $M_{\mathbb{R}^{d}}$, we deduce that, for all sets $B \in \mathcal{M}_{\mathcal{B}^{d}}$ of finite Q-measure, $\mathrm{Q}\left(B \cap \theta_{t}^{-1} B\right) \rightarrow 0$ as $\|t\| \rightarrow \infty$. This implies that $\sigma$ and then $\sum_{k=1}^{\infty}(1 / k !) \sigma^{* k}$ are Rajchman, which proves that $\left(M_{M_{\mathbb{R}^{d}}}, \mathcal{M}_{\mathcal{M}_{\mathbb{B}^{d}}}, \mathcal{P}_{\mathrm{Q}},\left\{\widetilde{\theta}_{t}\right\}_{t \in \mathbb{R}^{d}}\right)$ is mixing, thanks to Theorem 2.1. Once again, the semiconjugation with $\left(M_{\mathbb{R}^{d}}, \mathcal{M}_{\mathcal{B}^{d}}, \mathrm{P},\left\{\theta_{t}\right\}_{t \in \mathbb{R}^{d}}\right)$ implies that this last system is also mixing, and this completes the proof.

Remark. The results also apply to general ID random measures, thanks to the following arguments. An ID random measure gives birth to a point process, the associated Cox process, which is also ID (see [4] for a precise definition and properties of Cox processes). The random measure and the Cox processes share the same ergodic behaviour (ergodicity, weak mixing, or mixing) and are simultaneously square integrable, and if $\Gamma$ is the Bartlett spectrum of the random measure, the Bartlett spectrum of the Cox process is equivalent to $\Gamma+\lambda$. The conclusions easily follow.

\section{Acknowledgement}

The author acknowledges the support of the French Agence Nationale de la Recherche (ANR), under grant ATLAS (JCJC06_137446) 'From Applications to Theory in Learning and Adaptive Statistics'. 


\section{References}

[1] Aaronson, J. (1997). An Introduction to Infinite Ergodic Theory. American Mathematical Society, Providence, RI.

[2] Brémaud, P. and Massoulié, L. (2001). Hawkes branching processes without ancestors. J. Appl. Prob. 38, 122-135.

[3] Cornfeld, I. P., Fomin, S. V. And Sină̌, Y. G. (1982). Ergodic Theory. Springer, New York.

[4] Daley, D. J. and Vere-Jones, D. (1988). An Introduction to the Theory of Point Processes. Springer, New York.

[5] Kallenberg, O. (1983). Random Measures. Academic Press, London.

[6] Krengel, U. and Sucheston, L. (1969). On mixing in infinite measure spaces. Z. Wahrscheinlichkeitsth. 13, 150-164.

[7] Matthes, K., Kerstan, J. And Mecke, J. (1978). Infinitely Divisible Point Processes. John Wiley, Chichester. 